\title{
ONE-STAGE REIMPLANTATION FOR INFECTED TOTAL KNEE ARTHROPLASTY
}

\author{
S. B. GÖKSAN, M. A. R. FREEMAN
}

From the Royal London Hospital, Whitechapel

\begin{abstract}
One-stage reimplantation for the salvage of infected total knee arthroplasty in 18 patients was reviewed at an average follow-up of five years. There had been one recurrence and one new infection, both in rheumatoid patients with another focus of infection. In four other patients the clinical result was impaired by pain after walking (2) and limited flexion (2).

Our results suggest that one-stage reimplantation is a reasonably reliable procedure for the management of a loose infected prosthesis.
\end{abstract}

Successful results are reported for the treatment of infected hip arthroplasties by one-stage reimplantation (Buchholz et al 1981). We have had poor results using arthrodesis for infected knee arthroplasties (Freeman, King and O'Riordan 1983) and therefore chose to treat such cases by a one-stage reimplantation procedure from 1979. Encouraged by our early results (Freeman et al 1985), we have continued to perform this procedure in all patients with loose infected total knee arthroplasty irrespective of the chronicity of the infection and the virulence of the infecting organism. We now describe our results in 18 patients with an average follow-up of five years.

\section{MATERIALS AND METHODS}

Between July 1979 and September 1989, 19 patients with infection after total knee arthroplasty were treated with one-stage reimplantation. One patient, who was known to be free of infection at his last follow-up two years before he died, was excluded from this study because detailed information could not be retrieved. The clinical data of the remaining 18 patients are summarised in Table I.

At the time of reimplantation, the patient's ages ranged from 42 to 74 years (mean 61.4); there were 12 women and six men. The initial diagnosis was rheumatoid

S. B. Göksan, MD, Research Fellow

M. A. R. Freeman, FRCS, Consultant Orthopaedic Surgeon

Royal London Hospital, Whitechapel, London E1 1BB, England.

Correspondence should be sent to Mr M. A. R. Freeman.

(C) 1992 British Editorial Society of Bone and Joint Surgery

$0301-620 X / 92 / 1272 \$ 2.00$

J Bone Joint Surg [Br] 1992; 74-B: 78-82 arthritis in ten patients, osteoarthritis in seven and posttraumatic arthritis in one. Sixteen patients were infected after their initial arthroplasty and two became infected after revision for aseptic loosening in which the femoral component was exchanged in one knee and both components in the other. In these two patients the last revision was considered the index arthroplasty.

Before their primary total knee replacement three patients had had an operation for the treatment of the initial diagnosis: one had a supracondylar osteotomy of the femur, one had combined femoral and tibial osteotomies, and one had a patellectomy for comminuted fracture of the patella. Six patients were on corticosteroids at the time of the index arthroplasty. Other potential risk factors were recorded for 13 patients (Table II). All the rheumatoid patients had an additional potential risk factor, of which another focus of infection (4) and skin necrosis (3) were the most common. A drainage procedure followed by antibiotics had been attempted (unsuccessfully) in eight cases before reimplantation.

At their index operations seven patients had the Imperial College-London Hospital (ICLH) prostheses, ten had the Freeman-Samuelson (FS) prostheses, and one patient had an Insall-Burstein prosthesis. In six patients all components were cemented; in another six patients one component was cemented; six patients had a cementless arthroplasty at their index operation. The prostheses used in 13 arthroplasties had no stem. In three of four arthroplasties with a stemmed prosthesis there was no cement around the stem but cement was used elsewhere at the interface; the fourth was cementless. The Insall-Burstein prosthesis had a short (standard) stem and was fully cemented. At reimplantation the joint was converted to an ICLH prosthesis in three and to an FS prosthesis in 15 patients. 
Table I. Clinical data and results of 18 one-stage reimplantations for infected knee arthroplasties

\begin{tabular}{|c|c|c|c|c|c|c|c|c|c|c|}
\hline Case & Age & $\begin{array}{l}\text { Initial } \\
\text { diagnosis }\end{array}$ & $\begin{array}{l}\text { Interval* } \\
\text { (months) }\end{array}$ & $\begin{array}{l}\text { Infecting } \\
\text { organism }\end{array}$ & $\begin{array}{l}\text { Hospital } \\
\text { stay (days) }\end{array}$ & $\begin{array}{l}\text { Follow-up } \\
\text { (years) }\end{array}$ & $\begin{array}{l}\text { Pain on } \\
\text { walkingt }\end{array}$ & $\begin{array}{l}\text { Walking } \\
\text { (minutes) }\end{array}$ & $\begin{array}{l}\text { Extension } \\
\text { deficit (deg) }\end{array}$ & $\begin{array}{l}\text { Total } \\
\text { flexion (deg) }\end{array}$ \\
\hline 1 & 61 & OA & 17 & Staphylococcus aureus & 33 & 2 & None & $>30$ & 0 & 95 \\
\hline 2 & 63 & RA & 21 & S. epidermidis & 26 & 10 & None & $10 t$ & 0 & 90 \\
\hline 3 & 51 & RA & 36 & S. epidermidis & 32 & 10 & None & $5 \ddagger$ & 0 & 100 \\
\hline 4 & 70 & $\mathbf{R A}$ & 2 & S. aureus & 30 & 5 & None & $>30$ & 10 & 85 \\
\hline 5 & 51 & RA & 8 & S. aureus & 35 & 10 & Mild & Indoors onlył & $\mathbf{0}$ & 90 \\
\hline 6 & 65 & $\mathbf{O A}$ & 3 & S. epidermidis & 25 & 7 & None & $>30$ & 5 & 95 \\
\hline 7 & 61 & RA & 48 & $S$, epidermidis & 27 & 7 & None & $>30$ & 0 & 90 \\
\hline 8 & 44 & RA & $\mathbf{0}$ & S. epidermidis & 26 & 6 & None & $10 \ddagger$ & $\mathbf{0}$ & 105 \\
\hline 9 & 62 & RA & 26 & No growth obtained & 34 & 7 & None & Indoors only $\ddagger$ & $\mathbf{0}$ & 30 \\
\hline 10 & 69 & OA & 15 & S. epidermidis & 21 & 5 & None & $>30$ & $\mathbf{0}$ & 110 \\
\hline 11 & 68 & PTA§ & $\mathbf{0}$ & S. aureus & 23 & 1 & None & $>30$ & 5 & 35 \\
\hline 12 & 64 & OA & 60 & No growth obtained & 29 & 4 & None & $10 \ddagger$ & 0 & 100 \\
\hline 13 & 65 & RA & $\mathbf{0}$ & S. aureus & 21 & 3 & None & $>30$ & 0 & 90 \\
\hline 14 & 69 & RA & 30 & $\begin{array}{l}\text { S. epidermidis and } \\
\text { S. aureus }\end{array}$ & 39 & 3 & None & $10 \ddagger$ & 20 & 75 \\
\hline 15 & 42 & $\mathbf{R A}$ & 6 & $\begin{array}{l}\text { S. aureus and anaerobic } \\
\text { streptococcus }\end{array}$ & 19 & 3 & None & $>30$ & $\mathbf{0}$ & 110 \\
\hline 16 & 56 & OA & 60 & S. epidermidis & 18 & 2 & Moderate & 20 & 0 & 70 \\
\hline 17 & 71 & OA & 3 & S. aureus & 20 & 2 & Moderate & 10 & 0 & 90 \\
\hline 18 & 74 & OA & 16 & S. epidermidis & 15 & 1 & Mild & $>30$ & 0 & 110 \\
\hline
\end{tabular}

* interval between index arthroplasty and diagnosis of infection

+ mild pain $=$ not interfering with activities and/or sleep. Does not require analgesics

moderate pain $=$ activities reduced and/or sleep disturbed. Controlled by mild analgesics

$\ddagger$ restriction not due to affected knee

$\S$ PTA = post-traumatic arthritis

Table II. Potential risk factors in 13 infected knees treated by one-stage reimplantation

\begin{tabular}{llc}
\hline & Number of knees & Case \\
\hline Skin necrosis & 3 & $4,8,15$ \\
Postoperative drainage & 2 & 11,13 \\
Haematoma & 2 & 5,9 \\
Superficial wound infection & 1 & 6 \\
Delayed wound healing & 1 & 3 \\
Urinary tract infection & 1 & 17 \\
Complication after subsequent surgery & & \\
$\quad$ Foot surgery with postoperative infection & 1 & 2 \\
$\quad$ Reinfection in the arthrodesed knee and curettage & 1 & 3 \\
Skin infection in the ipsilateral foot & 1 & 14 \\
Histologically suspected infection at index arthroplasty & 1 & 7 \\
\hline
\end{tabular}

The average time between the index arthroplasty and the diagnosis of infection was 19.5 months (maximum 60). In three patients the infection occurred immediately postoperatively. Eight patients presented with an infection within a year of the index operation (early infection) and they all had a peri-operative potential risk factor; ten patients presented after the first year of index arthroplasty, and were recorded as having late infection (see Fig. 1).

Pain was the only constant and early symptom in all patients; in addition, seven had draining sinuses. Sixteen of the 18 patients gave a positive intra-operative culture 


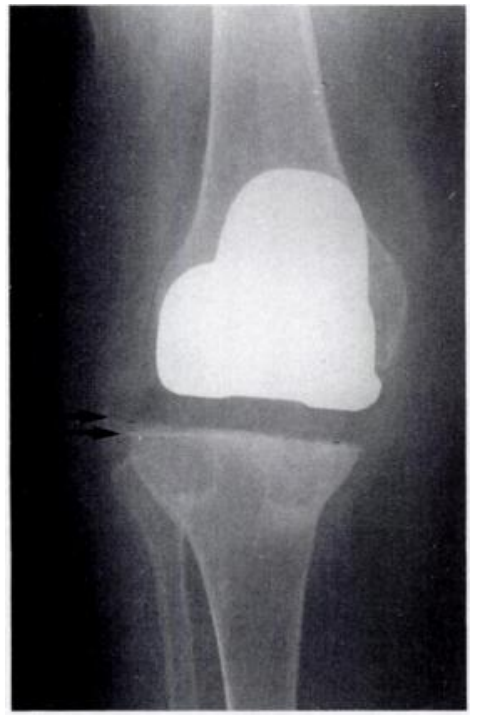

Fig. 1a

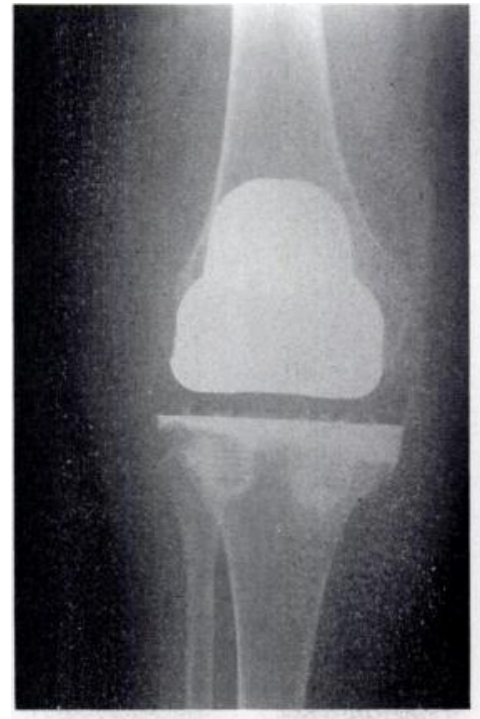

Fig. 1b

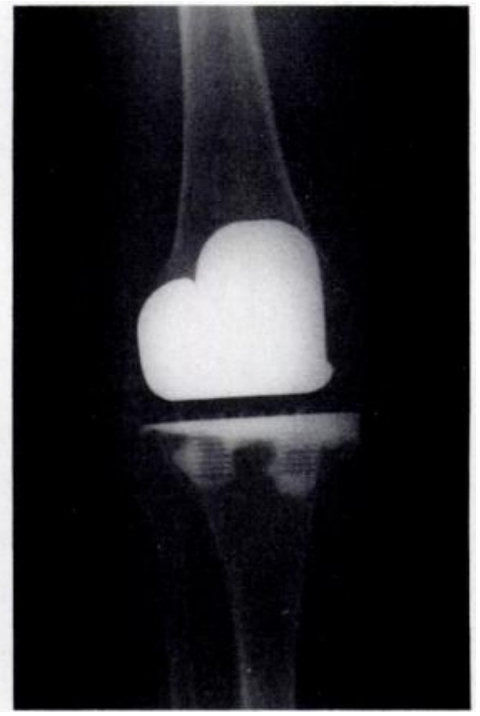

Fig. 1c

Case 2. A 63-year-old woman with rheumatoid arthritis. Figure 1a-Radiograph of the infected total knee arthroplasty two years after the index operation. The medial tibial condyle has collapsed allowing the prosthesis to lift-off laterally (arrowed). Figure $1 \mathrm{~b}$ - Immediately after one-stage reimplantation. Figure lc - Nine years after one-stage reimplantation.

combined with clinical and/or radiological signs of infection. The two patients with negative intra-operative cultures were included because they had draining sinuses from the knee confirmed by sinography and the operative findings were those of infection. Pre-operative courses of antibiotics were thought to explain the negative cultures.

Infecting organisms were Staphylococcus epidermidis in eight patients and Staphylococcus aureus in six. More than one organism was identified in two patients; Staphylococcus epidermidis and Staphylococcus aureus in one, Staphylococcus aureus and an anaerobic streptococcus in the other. No gram-negative organisms were identified.

Pre-operatively one or both components were seen to be loose on radiographs in 16 patients. At operation one or both components of 17 knees were loose. In one knee neither component was loose.

Operative procedure. Having approached the knee through the old incision, swabs and tissue samples were taken from the joint. The components and cement were removed and sent for culture. The interface membranes were taken for both bacteriological and histological examination. The joint was then thoroughly debrided, excising all tissue of doubtful viability. The joint was washed out with copious quantities of normal saline, and then packed with Povidone-iodine soaked swabs. The wound edges were approximated with a few sutures, antibiotics were administered intravenously, and the tourniquet was deflated for half an hour.

The operating team changed gowns and gloves, the patient was redraped and a new set of instruments was brought in for the next stage of the operation. The tourniquet was reinflated and the joint was again washed with normal saline. Culture swabs were again taken from the bone surfaces. The new implants were fixed with gentamicin-impregnated polymethylmethacrylate (Palacos, Schering Plough, Mildenhall, England). No cement was used around the stems which were dusted with antibiotic powder. Two suction drains were placed into the joint and the wound was closed in layers using nonabsorbable deep sutures and nylon for the skin. The knee was splinted until healing was assured and then flexion was started. The sutures were removed on the twelfth postoperative day.

The antibiotics, at first given intravenously, were chosen by assuming that the infecting organism was Staphylococcus aureus or on the basis of known bacteriology. This regime was changed, if necessary, when the results of the operative culture and antibiotic sensitivity studies were available and the oral route was used after one to two weeks. The duration of antibiotic therapy was three months.

The average hospital stay was 26 days (range 15 to 39). The patients were followed at regular intervals and evaluated clinically and radiographically both for evidence of infection and function of the prosthesis. The average length of follow-up was five years (one to ten). Apart from the patient who was excluded from this study, one patient died from lung cancer three years after reimplantation. He is included since there was no evidence of infection at his last follow-up, two years after the operation.

\section{RESULTS}

Recurrence of infection. There were two subsequent infections. One was in a 51-year-old woman with severe rheumatoid arthritis (case 5). She had had arthrodesis of 
her right, contralateral knee for another infected arthroplasty, and had suffered several recurrences of infection in this arthrodesis.

Seven months after the exchange arthifoptisting on her left knee the infection recurred with the same organism, and she was successfully treated by drainage and antibiotics. Although she had several subsequent episodes of sepsis in other joints and a recurrent urinary tract infection, the knee treated with reimplantation (and subsequently by drainage and antibiotics) presented no evidence of infection in the following nine years.

The other recurrent infection was in a 62-year-old woman with severe rheumatoid arthritis (case 9). No organism had been identified at the time of reimplantation, and the subsequent infection, six years later, was regarded as 'new' because it had developed concurrently with an infected varicose ulcer on the ipsilateral ankle. However, the connection was not proven bacteriologically: clinically the ulcer showed a streptococcal cellulitis but there was a Staphylococcus epidermidis bacteraemia, and a pseudomonas organism was grown from specimens taken from about the prosthesis. This infection settled with antibiotics, but the patient later developed an infection in a total hip replacement.

Clinical results. The average knee flexion on review was $87^{\circ}$ (Table I). Of the two patients with the least flexion $\left(30^{\circ}\right.$ and $\left.35^{\circ}\right)$, one had had a subsequent 'new' infection (case 9) and one a previous patellectomy. Four patients had a fixed flexion deformity ranging from $5^{\circ}$ to $20^{\circ}$. The patient who had a subsequent 'new' infection (case 9) had a $30^{\circ}$ extension lag as part of generalised muscle weakness at the time of the latest follow-up. He was, however, able to stand and walk with a frame by locking the knee in extension.

Walking was pain free and unlimited in eight patients. In five patients walking was limited, not by pain but by multiple joint involvement, and in one it was affected by cardiovascular disease. In the patient who had a 'new' infection (case 9), walking was limited due to generalised muscle weakness following prolonged immobilisation. Two patients had mild pain when walking. One of these could walk unlimited distances, but the other was confined indoors because of multiple joint involvement.

Two patients had pain in the knee severe enough to limit walking. In one of them pain was localised to the anterolateral aspect of the knee and restricted walking to ten minutes; this was the only patient who had (mild) rest pain. Further studies in this patient did not suggest infection and the cause of the pain has not been determined. In the second patient walking was limited to 20 minutes by anterior shin pain. This was thought to be due to contact between the tip of the stem of the prosthesis and the tibial cortex.

Walking aids were not required by seven patients; eight patients used them for reasons other than the knee treated by reimplantation. Six of these patients used either one stick or one crutch and one used two crutches. The patient who had a 'new' infection (case 9) used a walking frame. Two patients used one stick out of doors because of moderate pain when walking. The patient with a previous patellectomy used one stick out of doors. Radiological results. There were no radiolucent lines in three patients; 14 had an incomplete radiolucent line of less than $1 \mathrm{~mm}$ beneath the tibial component. The patient with recurrent infection (case 5) had a radiolucent line of less than $2 \mathrm{~mm}$ at the tibial bone-cement interface; this had developed in the first year after reimplantation and showed no progress in subsequent years. Two patients appeared to have a radiolucent line affecting less than $20 \%$ of the interface and $1 \mathrm{~mm}$ in thickness about the femoral component. Radiolucency about the femoral component is difficult to identify and evaluate.

The erythrocyte sedimentation rate was not helpful in diagnosis or follow-up because ten of our patients had rheumatoid arthritis. We have only recently been using serial C-reactive protein (CRP) levels as a laboratory aid.

\section{DISCUSSION}

Although infection is a relatively uncommon complication of total knee arthroplasty it is a serious one. In our early experience, attempted arthrodesis was the treatment of choice but, apart from the difficulty of obtaining solid fusion, the functional outcome was less than satisfactory, especially in patients with multiple joint involvement (Morrey et al 1989; Lettin et al 1990).

Rand and Bryan (1983) reported the successful eradication of infection in $57 \%$ of their series of 14 cases, with a clinical success rate of $35 \%$ after a delayed exchange procedure within 14 days of removal of a prosthesis. They did not use antibiotic-impregnated cement or long-term antibiotic therapy. Insall, Thompson and Brause (1983) used a two-stage procedure for reimplantation, with a six-week interval and intravenous antibiotic therapy between procedures. They were successful in eradicating the infection in all of their 11 cases. Most subsequent reports presented successful results and favoured two-stage reimplantation (Rosenberg et al 1988; Booth and Lotke 1989; Wilson, Kelley and Thornhill 1990; Windsor et al 1990). However, it was also clear that the procedure required a long period in hospital, and the patient had to be robust enough to withstand two or more operations.

One-stage reimplantation and reimplantation after a short delay have been considered to be unreliable (Johnson and Bannister 1986; Borden and Gearen 1987; Jacobs et al 1989), despite some reports of success in small groups of patients treated with various protocols (Walker and Schurman 1984; Grogan et al 1986; Borden and Gearen 1987; Teeny et al 1990). Borden and Gearen (1987) reported success in all three patients treated with one-stage reimplantation for gram-positive infections. Teeny et al (1990) presented one patient successfully 
treated with one-stage reimplantation. A recent study from Sweden reported 385 infected knee arthroplasties treated in various ways; with a mean follow-up of six years, no differences were found in the outcome of onestage as against two-stage procedures (Bengtson 1990).

In contrast with the literature concerning the knee, there have been several reports of one-stage reimplantation for infected total hip arthroplasties. Buchholz et al (1981) reported a $77 \%$ success rate in eradicating infection from infected hip arthroplasties, while subsequent reports gave success rates reaching $91 \%$ (Wroblewski 1986). Onestage reimplantation has therefore been recommended as the treatment of choice especially in properly selected patients with gram-positive infection.

We have succeeded in eradicating infection to date in 17 of 18 patients, all of whom were infected by grampositive organisms. The patient who had a recurrent infection (case 5) and the patient who had a 'new' infection (case 9) both suffered from advanced and severe rheumatoid arthritis and were clinically immunosuppressed. Nevertheless, neither of them required further major surgery; their infections settled with drainage and antibiotics in one case, and antibiotics only in the other. It has been reported that patients treated with two-stage procedures, with severe rheumatoid arthritis and suppression of the immune system, also have a high incidence of subsequent infection (Windsor et al 1990).

Eradication of infection is the main goal in the treatment of infected total knee arthroplasties but evaluation of the clinical results is also important in determining the success of a treatment. However, limitation of function due to multiple joint involvement in patients with severe rheumatoid arthritis makes it difficult to compare clinical series. Wilson et al (1990) have referred to this difficulty. In our series seven patients had limited function, due to multiple joint involvement in six patients and to cardiovascular disease in one. Apart from two patients with moderate pain on walking and two patients with limited flexion, none required analgesia for the knee. All patients could walk without limit imposed by the knee and had flexion in excess of $70^{\circ}$.

Our experience suggests that aggressive debridement, use of antibiotic-impregnated cement, and three months antibiotic therapy are important factors. Our patients for one-stage reimplantation were all infected with gram-positive organisms and none showed gross inflammatory signs nor systemic toxicity. In this group of patients one-stage exchange appears to be as reliable as two-stage procedures. Clearly one-stage reimplantation has the advantage of avoiding the added morbidity of a further operation and a long stay in hospital.

One or more of the authors have received or will receive benefits for personal or professional use from a commercial party related directly or indirectly to the subject of this article. In addition benefits have also been or will be directed to a research fund, foundation, educational institution, or other non-profit institution with which one or more of the authors is associated.

\section{REFERENCES}

Bengtson S. The infected knee arthroplasty. Doctoral dissertation. Lund, 1990.

Booth RE, Lotke PA. The results of spacer block technique in revision of infected total knee arthroplasty. Clin Orthop 1989; 248:57-60.

Borden LS, Gearen PF. Infected total knee arthroplasty: a protocol for management. J Arthroplasty 1987; 2:27-36.

Buchholz HW, Elson RA, Engelbrecht E, et al. Management of deep infection of total hip replacement. J Bone Joint Surg [ Br] 1981; 63 B:342-53.

Freeman MAR, King JB, O'Riordan SM. The day frame. In: Ackroyd $\mathrm{CE}, \mathrm{O}$ Connor BT, de Bruyn PF, eds. The severely injured limb. Edinburgh, etc: Churchill Livingstone, 1983:129-43.

Freeman MAR, Sudlow RA, Casewell MW, Radclif SS. The management of infected total knee replacements. J Bone Joint Surg [Br] $1985 ; 67-B: 764-8$.

Grogan TJ, Dorey F, Rollins J, Amstutz HC. Deep sepsis following total knee arthroplasty: ten-year experience at the University of California at Los Angeles Medical Center. J Bone Joint Surg [Am] 1986 ; 68-A :226-34.

Insall JN, Thompson FM, Brause BD. Two-stage reimplantation for the salvage of infected total knee arthroplasty. J Bone Joint Surg [Am] $1983 ; 65-A: 1087-98$.

Jacobs MA, Hungerford DS, Krackow KA, Lennox DW. Revision of septic total knee arthroplasty. Clin Orthop 1989; 238:159-66.
Johnson DP, Bannister GC. The outcome of infected arthroplasty of the knee. J Bone Joint Surg [Br] 1986; 68-B:289-91.

Lettin AWF, Neil MJ, Citron ND, August A. Excision arthroplasty for infected constrained total knee replacements. J Bone Joint Surg [Br] 1990; 72-B:220-4.

Morrey BF, Westholm F, Schoifet S, Rand JA, Bryan RS. Long-term results of various treatment options for infected total knee arthroplasty. Clin Orthop 1989; 248:120-8.

Rand JA, Bryan BS. Reimplantation for the salvage of an infected total knee arthroplasty. J Bone Joint Surg [Am] 1983; 65-A:1081-6.

Rosenberg AG, Haas B, Barden R, et al. Salvage of infected total knee arthroplasty. Clin Orthop 1988; 226:29-33.

Teeny SM, Dorr L, Murata G, Conaty P. Treatment of infected total knee arthroplasty. J Arthroplasty 1990; 5:35-9.

Walker RH, Schurman DJ. Management of infected total knee arthroplasties. Clin Orthop 1984; $186: 81-9$.

Wilson MG, Kelley K, Thornhill TS. Infection as a complication of total knee replacement arthroplasty: risk factors and treatment in sixtyseven cases. J Bone Joint Surg [Am] 1990; 72-A :878-83.

Windsor RE, Insall JN, Urs WK, Miller DV, Brause BD. Two-stage reimplantation for the salvage of total knee arthroplasty complicated by infection. J Bone Joint Surg [Am] 1990; 72-A :272-8.

Wroblewskd BM. One-stage revision of infected cemented total hip arthroplasty. Clin Orthop 1986; $211: 103-7$ 